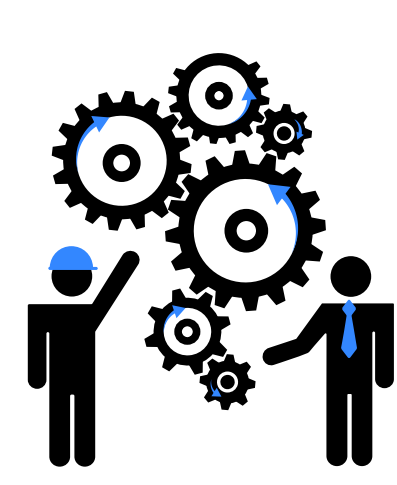

\title{
DISEÑO DE LA FAJA DE TRANSPORTE Y SELECCION DEL EJE PARA EL PROCESO DE CORTE DE AJÍ JALAPEÑO
}

\author{
DESIGN OF THE CONVEYING SYSTEM AND AXIS SELECTION \\ FOR AJÍ GALAPEÑO CUTTING PROCESS
}

\author{
Roberto Belarmino Quispe Cabana ${ }^{1}$ \\ 1 Ingeniero Mecánico, Universidad Continental. \\ E mail: rquispe@continental.edu.pe
}

\section{Resumen}

Las empresas del sector agroindustrial usualmente realizan el corte de forma manual (capacidad de $15 \mathrm{~kg} / \mathrm{h}$ por persona y utilizan de 60 a 70 personas en sus líneas de producción) o con maquinaria funcional que existe en el mercado, con diseños que no satisfacen los requerimientos de las empresas, pues generan un alto porcentaje de descarte (de $25 \%$ a $35 \%$ ) y una capacidad operativa de $600 \mathrm{~kg} / \mathrm{h}$. Debido a que el tamaño del ají jalapeño es irregular y la deflexión que pueda generar el eje podría repercutir en el corte, se plantea como objetivo reducir el descarte o disminuir la merma (menor a $8 \%$ ) mediante un adecuado posicionamiento de la faja transportadora y selección del diámetro del eje. El material utilizado en la construcción es de acero inoxidable para el sistema de alimentación, transporte, corte y descarga, por el contacto que tienen los elementos mecánicos de estos sistemas, entre ellos el sistema de corte con el ají jalapeño. Para lograr un corte adecuado (por mitad), la velocidad, tanto del sistema de transporte como para el corte, se regula con un variador de velocidad. Con el adecuado posicionamiento de las fajas transportadoras en ángulo de $45^{\circ}$ y una correcta selección del diámetro para soportar el esfuerzo de torsión y momento flector se logra reducir la merma por descarte a menos del $8 \%$, es decir a 3,4 \%; bajo estos parámetros se logra una producción total promedio de $1584 \mathrm{~kg} / \mathrm{h}$ y una producción neta de $1583 \mathrm{~kg} / \mathrm{h}$.

\begin{abstract}
The agroindustrial companies usually perform the cut manually (capacity of $15 \mathrm{~kg} / \mathrm{h}$ per person, using 60 - 70 people in their production lines) or with functional machinery that exists in the market with designs that do not match the requirements of the companies. This matter generates a high percentage of discarding (from 25 to 35 $\%$ ) and operative capacity of $600 \mathrm{~kg} / \mathrm{h}$.

Because the size of the jalapeño pepper is irregular and the deflection that the shaft can generate can have an impact on the cut, the objective is to reduce the discarding or reduction of less than $8 \%$ by means of an adequate positioning of the conveyor belt, selection of the diameter of the axis.

The material used in the construction is stainless steel for the feeding, transport, cutting and unloading system; by the contact that the mechanical elements of these systems have, including the cutting system with the jalapeño pepper. The speed variator regulated the speed, both for the speed of the transport system and cutting, to achieve a proper cut (by half). With the proper positioning of the conveyor belts at a $45^{\circ}$ angle and a correct diameter selection to withstand the torsional stress and moment of bending, it is possible to reduce the waste by discarding at less than $8 \%$, that is, at $3.4 \%$. Under these parameters, was achieved a total production of $1584 \mathrm{~kg} / \mathrm{h}$ with a net production of $1583 \mathrm{~kg} / \mathrm{h}$.
\end{abstract}

Keywords: Angle; jalapeño pepper; cut; axis.

Palabras clave: Ángulo; ají jalapeño; corte; eje. 


\section{INTRODUCCIÓN}

De acuerdo con información proporcionada por el gremio exportador, solo el 3,6 \% de nuestras exportaciones contienen una tecnología mediana y alta. En tanto, en Chile este porcentaje asciende a 5,3\%, en Colombia a 10,3\% y en México a 66,1 \% [1]. Las empresas que fabrican máquinas agroindustriales en el ámbito local y nacional son pocas e incluso sus productos o máquinas no tienen la calidad para ser vendidos a mayor escala a nivel local o nacional. Las máquinas agroindustriales del medio en su mayoría son adquiridas por importación.

El ají jalapeño (conocido también como chile) pertenece al género Capsicum; el fruto donde se encuentran las semillas es una baya hueca carnosa o semicartilaginosa, puede alcanzar distintos tamaños, desde poco menos de $1 \mathrm{~cm}$ hasta $30 \mathrm{~cm}$ de largo, y su forma va de lo redondo a lo alargado, en colores que oscilan de distintos tonos de amarillo y verde, en estado inmaduro, a rojo y hasta café al madurar; es también un alimento de gran valor nutritivo. Se considera que es el vegetal con mayor concentración de ácido ascórbico; en fresco contiene más del doble de vitamina $\mathrm{C}$ que el limón y la naranja y casi seis veces más que la toronja; en seco, por su parte, contiene vitamina A en una proporción mayor que las zanahorias, por ejemplo. Asimismo, los chiles (ajíes jalapeños) poseen cantidades significativas, aunque menores, de vitaminas E y B, y de algunos minerales [2].

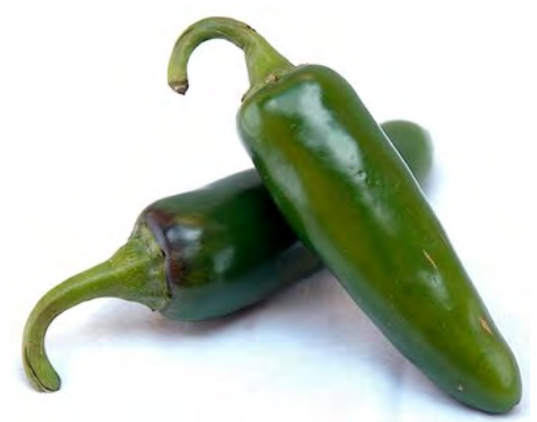

Figura 1. Ají jalapeño (Foto: captura de Youtube)

Tradicionalmente, el principal proveedor de ají jalapeño al mercado estadounidense ha sido México. Sin embargo, se observa que otros países han empezado a ganar terreno en el mercado, entre otros destacan India, China, El Salvador y Perú. Entre las principales empresas peruanas exportadoras del ají jalapeño destaca Agro Mantaro.

El objetivo es reducir las mermas producto de un mal corte en el ají jalapeño a través de un análisis del sistema de corte; merma por descarte se considera a aquellos cortes realizados que no son por la mitad del ají y repercuten en la producción neta, en su respectiva presentación y en el proceso para su venta posterior. Para ello, se analizan diversos principios de cortes y de diseño, para luego plantear el diagrama funcional, una matriz morfológica, evaluación y presentación de la alternativa de prototipo para su respectiva fabricación.

Se evalúa el funcionamiento de la máquina en vacío, así como bajo carga de ají jalapeños; se observa, asimismo, la calidad de corte que desarrolla; se mide la producción y el porcentaje de merma que genera.

\section{DISEÑO CONCEPTUAL}

Estudio comparativo de principios de funcionamiento

- Principio de funcionamiento de máquina cortadora-forma de rodajas

El producto se entrega a dos cintas de alimentación de alta velocidad (1) dispuestas en forma de V (Figura 2). Las correas están sincronizadas con la rueda rebanadora giratoria (2) para garantizar el avance adecuado del producto por revolución de la rueda. Las cuchillas (3) bajo tensión sirven como radios y sostienen el aro de la rueda rebanadora; están ligeramente inclinadas para crear un ángulo uniforme entre el cubo y el aro (Figura 2).

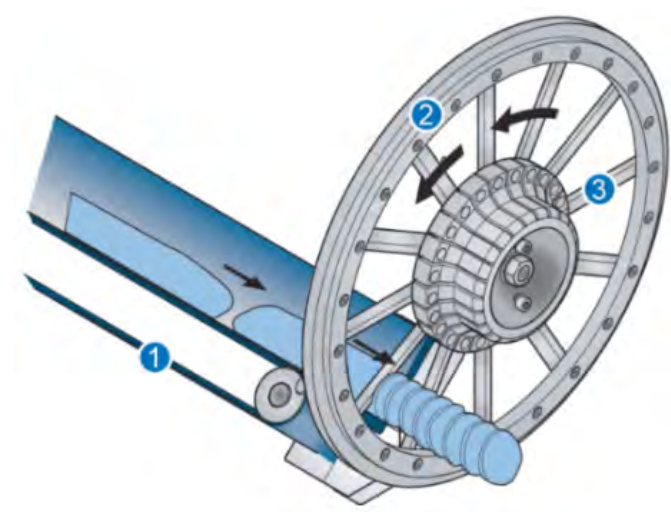

Figura 2. Principio de corte por rodajes y disposición de faja transportadora . Fuente: URSHEL ${ }^{\circledast}[3]$

- Principio de funcionamiento de máquina cortadora-forma de rodajas

Consta de un gabinete con doble puerta de acceso para ajustes y producto, un sistema de cuchillas y bases con ruedas de altura ajusta. Su diseño 
permite una fácil y rápida limpieza del equipo; su capacidad es de 75 a $100 \mathrm{~kg} / \mathrm{h}$ (depende de la habilidad del operario) y la alimentación es manual. Las dimensiones son largo total: $900 \mathrm{~mm}$, ancho total: $906 \mathrm{~mm}$, altura total: $1100 \mathrm{~mm}$, altura de descarga: $525 \mathrm{~mm}$ (Figura 3).
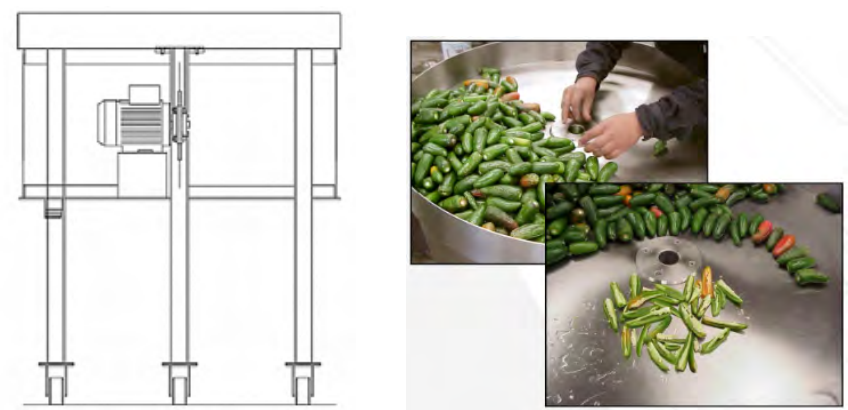

Figura 3. Principio de corte a mitad de ají jalapeño

\section{DISEÑO FUNCIONAL}

\section{Caja negra}

El ají jalapeño previamente lavado y sin el pedúnculo es transportado en la máquina para ser cortado por mitad, y por supuesto a la salida hay dos conductos, uno para el fruto partido por mitad y otro para derivar el olor (Figura 4).

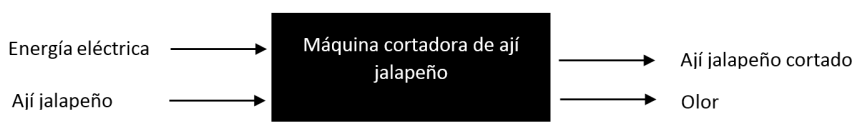

Figura 4. Caja negra

\section{Estructura funcional}

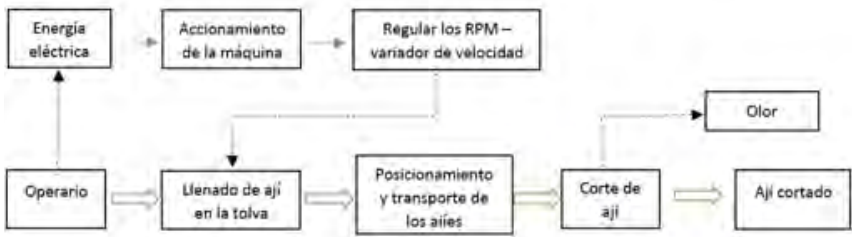

Figura 5. Estructura funcional

El flujo del esquema empieza por activar el sistema de alimentación de energía eléctrica, que acciona el motor del sistema de transmisión y de corte; en ese instante comienza el llenado del ají a la tolva, y es transportado por una faja transportadora hacia el sistema de corte; el fruto cortado pasa a un recipiente y el olor del picor se deriva hacia el ambiente por la parte superior (Figura 5).

\section{Matriz morfológica}

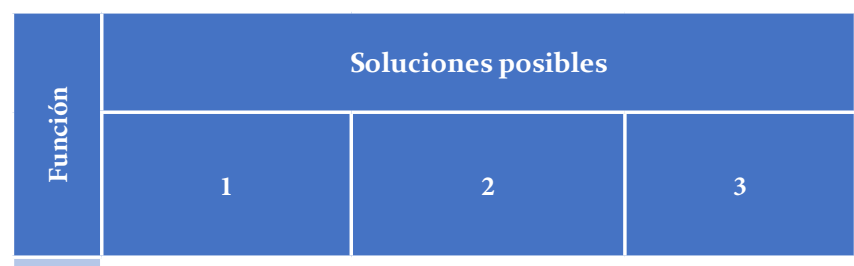

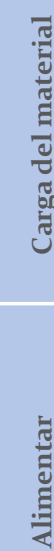
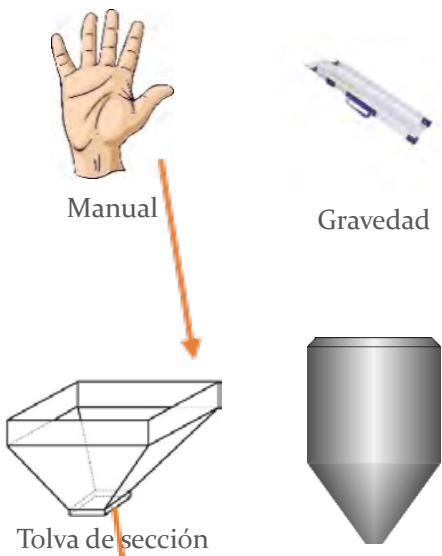

Gravedad

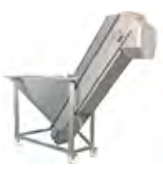

Faja

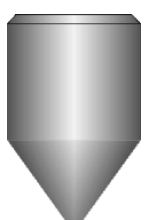

Tolva de sección circular

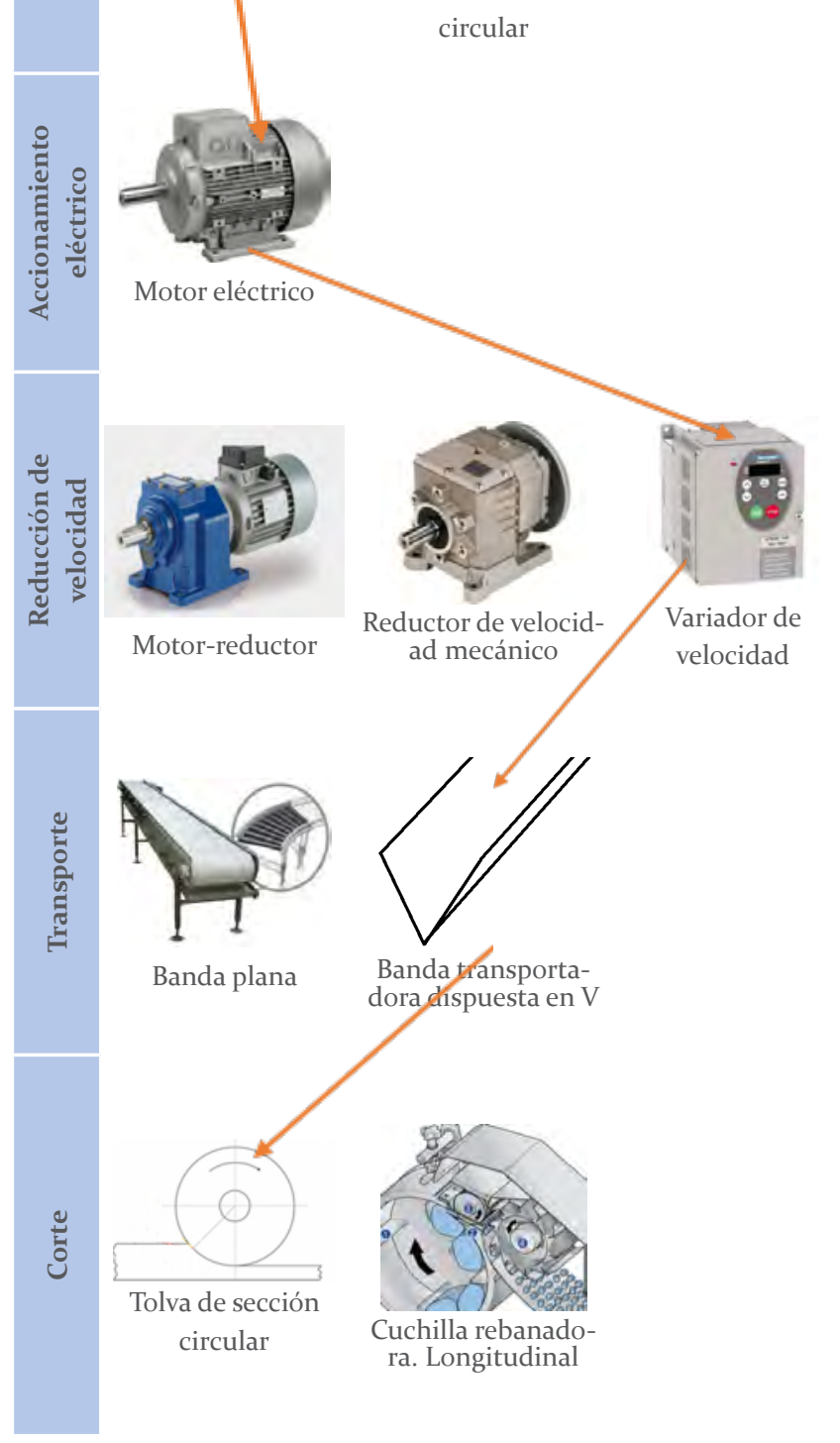


Alternativa de solución
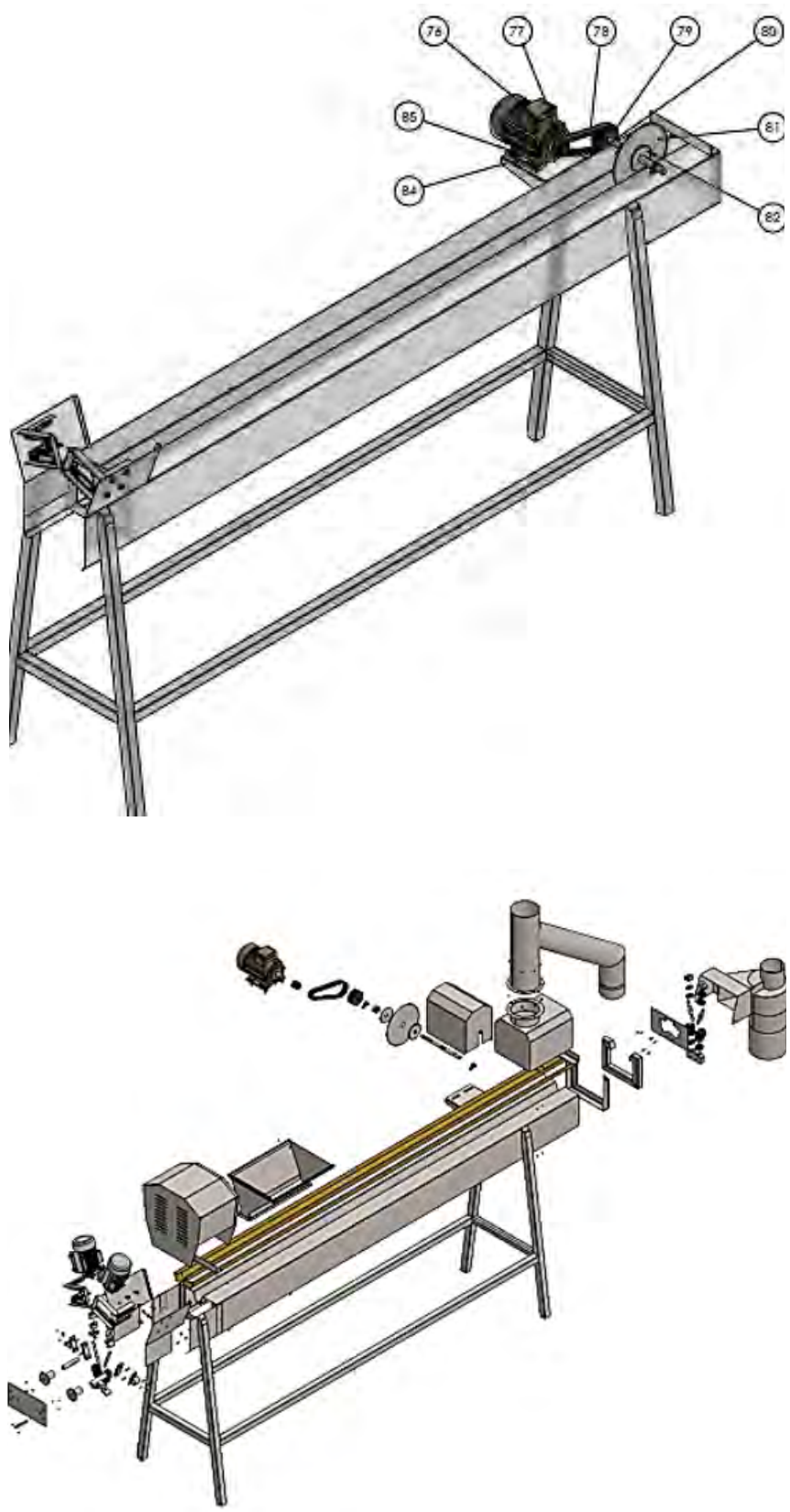

Figura 6. Presentación de sistema de corte y (en despiece) de propuesta final. Fuente: Elaboración propia

\section{ANÁLISIS DE DETALLE}

Ángulo de posición de banda transportadora para ají jalapeño

Para disponer el ángulo de inclinación y la faja de transportadora, se determina el tamaño promedio de los ajíes jalapeños, según los datos asignados en la Tabla 1.
Tabla 1. Dimensión promedio de ají jalapeño

\begin{tabular}{|l|l|l|}
\hline \multirow{2}{*}{ Diámetro } & \multicolumn{3}{|c|}{ Tamaño pequeño } \\
\hline \multirow{2}{*}{ Mínimo } & $20 \mathrm{~mm}$ \\
\cline { 2 - 3 } & Máximo & $30 \mathrm{~mm}$ \\
\hline \multirow{2}{*}{ Longitud } & Mínimo & $40 \mathrm{~mm}$ \\
\cline { 2 - 3 } & Máximo & $65 \mathrm{~mm}$ \\
\hline \multirow{2}{*}{ Diámetro } & Tamaño grande & \\
\cline { 2 - 3 } & Mínimo & $22 \mathrm{~mm}$ \\
\hline \multirow{2}{*}{ Longitud } & Máximo & $30 \mathrm{~mm}$ \\
\hline & Mínimo & $54 \mathrm{~mm}$ \\
\hline & Máximo & $75 \mathrm{~mm}$ \\
\hline
\end{tabular}

Fuente: Elaboración propia

En la Figura 7 se muestra el ángulo máximo y mínimo de acuerdo con el tamaño de los ajíes jalapeños. De aquí se obtiene la fijación del ángulo de la faja transportadora, que es de $47,92^{\circ}$ aproximadamente; el ángulo para la fijación es de $45^{\circ}$.
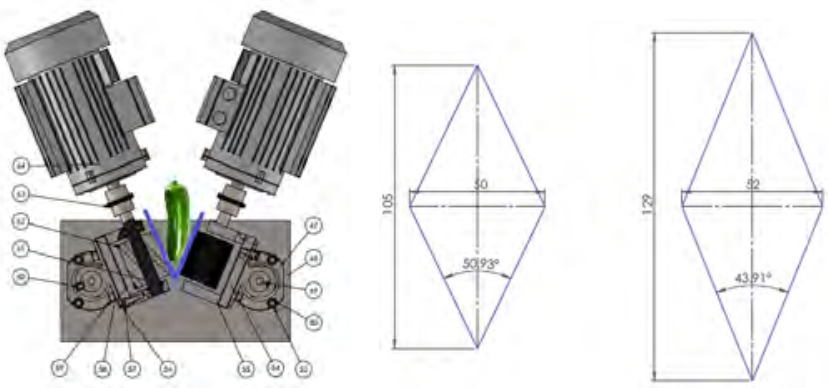

Figura 7. Ángulo promedio de la disposición de faja transportadora. Fuente: Elaboración propia

\section{Selección del material}

Para elegir el mejor material, la selección debe comprender las consideraciones de disponibilidad, costo total, propiedades del material y los procesos de manufactura aplicando la experiencia, el conocimiento experto de ingeniería, índice de selección y el conocimiento de los posibles modos de falla [3].

El tipo de acero a seleccionar es un acero austenítico inoxidable 304', elegido por su aplicación para equipos químicos y tuberías, componentes de intercambiadores térmicos, equipos y utensilios de manipulación de lácteos y alimentos, recipientes y componentes criogénicos, aplicaciones arquitectónicas y estructurales expuestas a atmósferas no marinas. 
Tabla 2. Propiedad a tensión del acero inoxidable 304

\begin{tabular}{|c|c|c|c|c|}
\multirow{2}{*}{ Material } & \multicolumn{2}{|c|}{ Resistencia ultima } & \multicolumn{2}{c|}{$\begin{array}{c}\text { Resistencia a la } \\
\text { cedencia }\end{array}$} \\
\cline { 2 - 5 } & $\mathbf{k l} /$ pulg $^{2}$ & $\mathbf{M P a}$ & $\mathbf{k l} /$ pulg $^{2}$ & $\mathbf{M P a}$ \\
\hline $\begin{array}{c}\text { Acero inoxidable } \\
304\end{array}$ & 83 & 572 & 40 & 276 \\
\hline
\end{tabular}

Fuente: Juvinall R. (2013, p. 782)

\section{Diámetro del eje del sistema de corte}

El eje es uno de los elementos motrices que va a estar sometido a esfuerzo flexionante y de corte; para dimensionar al eje, se utiliza la teoría de relación elíptica ASME. El criterio ASME elíptico también toma en cuenta la fluencia, pero no es completamente conservador a lo largo de todo su rango [4].

a) Momento flector máximo en el eje

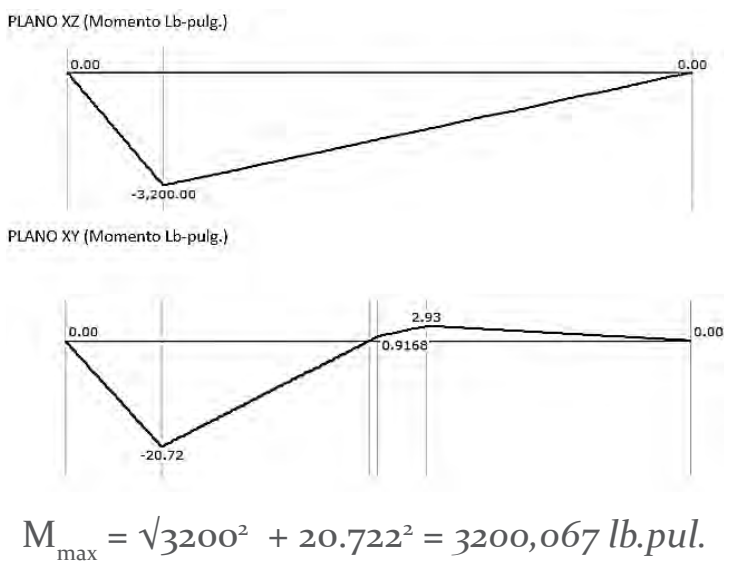

b) Torsión

$$
\begin{aligned}
& T=63025 H_{\text {nom }} K_{s} n_{d} / n \\
& =63025(2)(1,5)(1,1) / 1750=\mathbf{1 1 8 , 8 4 7} \mathrm{lb} . p u l g
\end{aligned}
$$

c) Diámetro del eje

Mediante la teoría de relación elíptica ASME, se realiza un análisis de falla por fatiga con la siguiente ecuación:

$$
d=\left\{\frac{16 n}{\pi}\left[4\left(\frac{K_{f} M_{a}}{S_{e}}\right)^{2}+3\left(\frac{k_{f_{s}} T_{m}}{S_{y}}\right)^{2}\right]^{1 / 2}\right\}^{1 / 3}
$$

$$
\begin{aligned}
& d=\left[\frac{16(2)}{3,14}\left[4\left(\frac{1,32 \times 3200,067}{50185}\right)^{2}+3\left(\frac{0,872 \times 118,847}{40000}\right)^{2}\right]\right] \\
& d=\left[\frac{16(2)}{3,14}\left[4\left(\frac{1,32 \times 3200,067}{50185}\right)^{2}+3\left(\frac{0,872 \times 118,847}{40000}\right)^{2}\right]^{1 / 2}\right]^{1 / 3}
\end{aligned}
$$

$$
d=1,19 \text { pulg. }
$$

\section{RESULTADO}

\section{Máquina}

La máquina es de accionamiento eléctrico y el material es de acero inoxidable; está regulada por variadores de velocidad para el sistema de transporte y de corte.

El producto cortado cae hacia abajo. Al realizar la prueba en vacío, la máquina no presentó problemas.

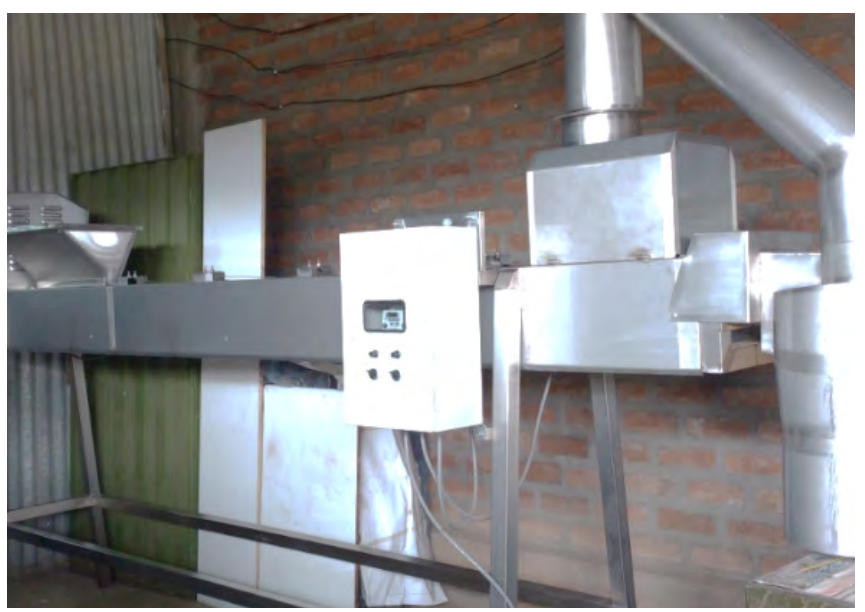

Figura 6. Máquina cortadora de ají jalapeño. Fuente: Cortesía Proyecto Innovate Perú.

\section{Producción}

La prueba bajo carga se realizó varias veces; el resultado que se plasma en la Tabla 3 genera menos producción en comparación con el resultado anterior; sin embargo, genera menos pérdida por descarte o mermas producidas por un mal corte que en promedio es de $3,4 \%$.

Tabla 3. Resultado con motor de corte a $60 \mathrm{~Hz}$ y el motor del sistema de transporte a $23 \mathrm{~Hz}$

\begin{tabular}{|c|c|c|c|c|c|c|}
\hline Pruebas & Cantidad & Tiempo & Merma (kg) & Merma (\%) & Producción total (kg/h) & Producción neta \\
\hline 1 & $11 \mathrm{~kg}$ & $17 \mathrm{~s}$ & 0,60 & 5,5 & 2329 & 2328 \\
\hline 2 & $11 \mathrm{~kg}$ & $17 \mathrm{~S}$ & 0,64 & 5,81 & 2329 & 2328 \\
\hline 3 & $11 \mathrm{~kg}$ & $17 \mathrm{~S}$ & 0,62 & 5.3 & 2329 & 2328 \\
\hline
\end{tabular}


Tabla 4. Resultado con motor de corte a $25 \mathrm{~Hz}$ y el motor que acciona la faja de transporte a $23 \mathrm{~Hz}$

\begin{tabular}{|c|c|c|c|c|c|c|}
\hline Pruebas & Cantidad & Tiempo & Merma $(\mathbf{K g})$ & Merma (\%) & Producción total kg/h) & Producción neta \\
\hline 1 & $11 \mathrm{~kg}$ & $25 \mathrm{~S}$ & 0,37 & 3,3 & 1584 & 1583 \\
\hline 2 & $11 \mathrm{~kg}$ & $25 \mathrm{~S}$ & 0,39 & 3,5 & 1584 & 1583 \\
\hline 3 & $11 \mathrm{~kg}$ & $25 \mathrm{~S}$ & 0,36 & 3,2 & 1584 & 1583 \\
\hline
\end{tabular}

\section{Corte}

La merma es mayor cuando la velocidad del disco de corte es a $60 \mathrm{~Hz}$ (manipulado con el variador de frecuencia); la merma aumenta por la misma velocidad lineal y centrifuga tanto al ingreso del disco de corte como al salir de ella.

Los cortes mejoran al manipular los motores eléctricos de accionamiento a una frecuencia de 23 a $25 \mathrm{Hzy}$ disminuyen la merma (cortes de ají que no pasan por el medio como resultado de la prueba, ver Figura 8).
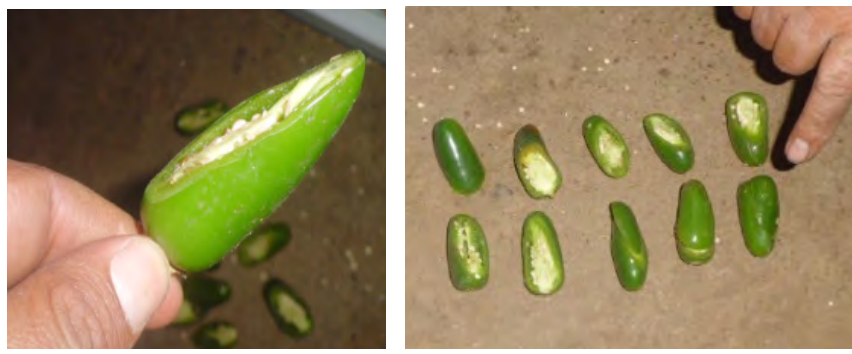

Figura 7. Separación por destacarte de cortes asimétricos
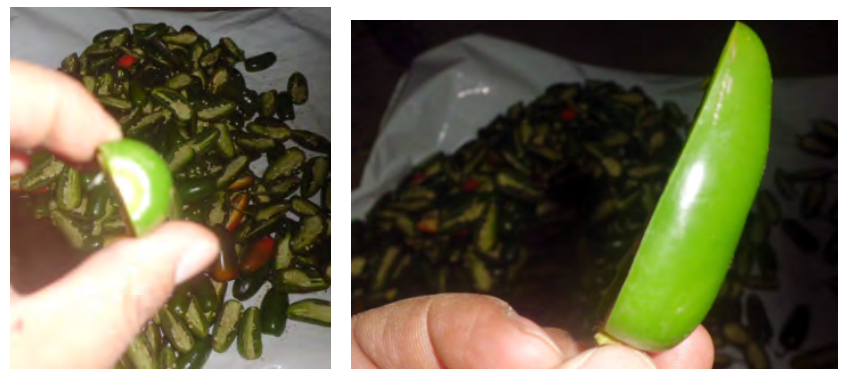

Figura 8. Muestra de un buen corte (por el medio).

\section{CONCLUSIONES}

- El ángulo de posicionamiento adecuado de la faja transportadora depende del tamaño de los ajíes jalapeños, para este caso es de $45^{\circ}$.

- El diámetro del eje del sistema de corte que garantiza soportar los esfuerzos a los que estará sometido es de 1,19 pulg (13/16 «) con un factor de seguridad de 2.

- El porcentaje de merma varía con la velocidad de producción, encontrándose que debería ser de 1583 $\mathrm{kg} / \mathrm{h}$ para tener una merma por descarte de $3,3 \%$.

\section{RECOMENDACIONES}

- Considerar la seguridad en la manipulación del sistema de corte de la máquina.

- Monitorear constantemente y realizar el mantenimiento previsto.

- Innovar en el sistema de corte para producir diferentes tipos de secciones en otros frutos a fin de multiplicar sus funciones.

\section{AGRADECIMIENTO}

A la Unidad de Investigación de la Facultad de Ingeniería de la Universidad Continental.

Al FYNCIT e Innovate Perú.

\section{REFERENCIAS}

[1] Diario Gestión (edición digital), Economía $<$ https://gestion.pe/economia/3-6-exportaciones-peruanas-contiene-tecnologia-mediana-alta-95664> [Consulta: Junio, 2015]

[2] Aguirre, E. y Muñoz, V. El chile como alimento. Revista de la Academia Mexicana de Ciencias 66 (3), (2015). [Consulta en línea]. Recuperado de http://www.revistaciencia.amc.edu.mx/images/revista/66_3/PDF/Chile.pdf

[3] URSHEL ${ }^{\circledast}$ Cortadora TranSlicer 2000 ${ }^{\circledast}$ Cutter Brochure https://es.urschel.com/sites/es.urschel.com/files/L2551SP_TRS2ofs.pdf [Consulta: Junio, 2015]

[4] Juvinall, R. Diseño de elementos de máquinas. 2. ${ }^{a}$ edición. México: Limusa-Wiley, 2013.

[5] Budynas, R. y J. Keith. Diseño en ingeniería mecánica de Shigley. México: Mc Graw Hill Interamericana, 2008. 